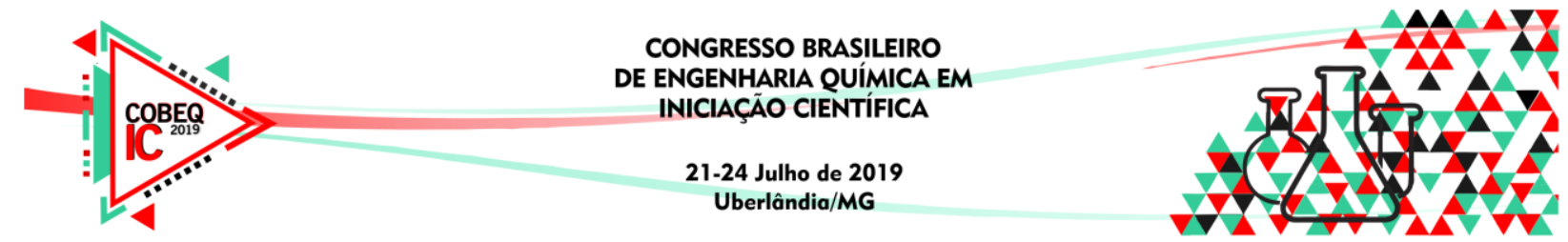

\title{
DESENVOLVIMENTO DE UMA BIBLIOTECA DE MODELOS PARA SIMULAÇÃO DE PROCESSOS ENVOLVENDO SÓLIDOS PARTICULADOS COM O SIMULADOR EMSO
}

\author{
L. A. SILVA ${ }^{1}$ e A. R. SECCHI ${ }^{2}$ \\ ${ }^{1}$ Universidade Federal do Rio de Janeiro, Escola de Química \\ ${ }^{2}$ Universidade Federal do Rio de Janeiro, PEQ-COPPE \\ E-mail para contato: lucasandrade@eq.ufrj.br; arge@ peq.coppe.ufrj.br
}

\begin{abstract}
RESUMO - Com o objetivo de suprir uma necessidade pouco explorada em simuladores de processos químicos, este trabalho traz à luz uma nova ferramenta para a área de sistemas particulados, através de uma biblioteca de modelos matemáticos para o simulador de processos EMSO. Foram implementados 47 modelos, contemplando correntes de processo, equipamentos separadores, cominuidores e equipamentos acessórios, juntamente à elaboração de ícones para sua representação em diagramas de processo. Os modelos foram testados individualmente e sua utilização foi ilustrada através de estudos de caso simples, análogos a processos industriais reais.
\end{abstract}

\section{INTRODUÇÃO}

Sólidos particulados estão presentes em uma infinidade de processos industriais de alta importância: processos orgânicos, no refino de petróleo e produção de polímeros; inorgânicos, no processamento de minérios; e até biotecnológicos, na produção de alimentos via fermentação, dentre outros. Essa variedade por si só já mostra o quanto o conhecimento dos sistemas particulados afeta o mundo moderno nas esferas econômica, energética e ambiental.

A simulação de diagramas de processo (PFDs) é talvez a mais importante ferramenta computacional para o engenheiro na análise, projeto e otimização do desempenho de plantas industriais. Muitos simuladores de processos estão disponíveis no mercado e na literatura, divididos entre modular sequenciais e orientados por equações. No primeiro tipo, as equações e algoritmos estão atrelados a cada bloco e os sistemas são resolvidos sequencialmente; já no segundo tipo, todas as equações que definem os modelos de cada bloco são resolvidas simultaneamente. Em geral, esses simuladores atendem bem aos requisitos da engenharia química clássica, tratando de processos envolvendo apenas fluidos, mas há muito espaço para desenvolvimento no que trata de sólidos particulados, área mais bem explorada por simuladores específicos da área de processamento mineral, que, apesar disso, pecam justamente no tratamento dos fluidos. Softwares comerciais dominam essa área.

A mais óbvia diferença entre a simulação de fluidos e de sólidos é a necessidade de designar atributos distribuídos para descrever os sólidos, enquanto fluidos estão completamente definidos ao se conhecer sua temperatura, pressão e composição. O mais importante desses atributos é o tamanho das partículas (Hartge et al., 2006), além de 


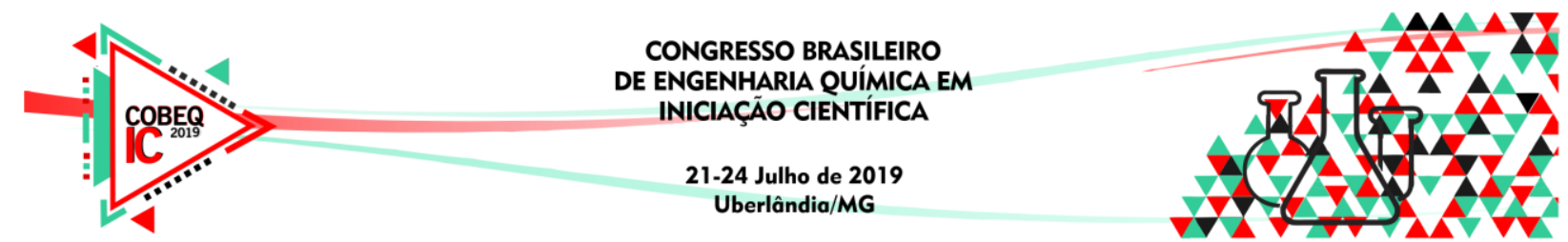

composição, forma e outros. Quando se trata de suspensões sólido-fluido, outras grandezas se tornam importantes também, como concentração mássica e volumétrica. A definição dessas grandezas é essencial para descrever as principais operações industriais que tratam sólidos.

O simulador EMSO (Environment for Modeling, Simulation and Optimization), introduzido por Soares e Secchi (2003), possui características que o destacam dos demais simuladores gratuitos, e mesmo da maioria dos comerciais. É do tipo orientado por equações e permite ao usuário criar e modificar modelos livremente através de uma linguagem de modelagem própria que é intuitiva e fácil de utilizar, baseando-se em duas entidades básicas chamadas Model e FlowSheet. A primeira é própria para descrever modelos individuais, como de cada equipamento; já a segunda é própria para descrever sistemas, fluxogramas, compostos por Models e suas conexões. O software oferece ainda a possibilidade de definir modelos como subtipos de outros modelos através de um conceito de herança.

Com isso, o objetivo deste trabalho é desenvolver uma biblioteca de modelos matemáticos de correntes e equipamentos para simulação de processos envolvendo sólidos particulados através do simulador EMSO, que não possui modelos nativos para tais sistemas.

\section{METODOLOGIA}

Foi realizada uma extensa busca bibliográfica acerca das características dos sistemas particulados e das suas operações unitárias, contemplando sistemas puramente sólidos e sólido-fluido. A Figura 1 mostra a metodologia adotada.

Figura 1 - Esquema da metodologia utilizada no desenvolvimento da biblioteca.

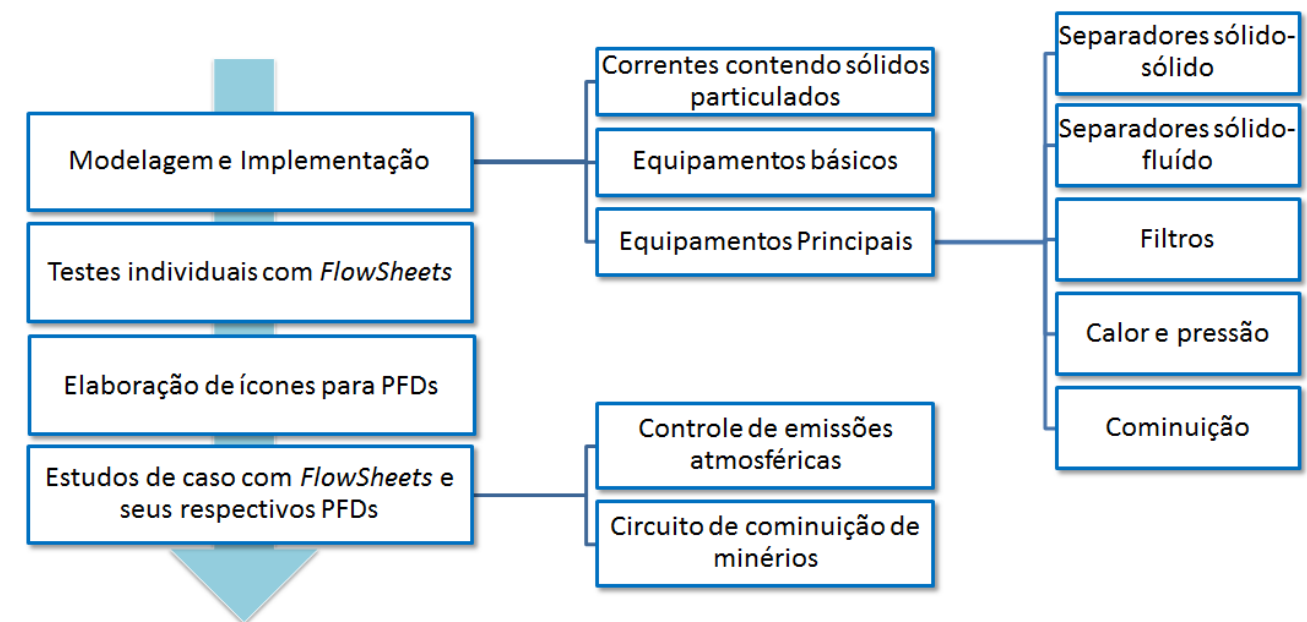

Foram tomadas as seguintes premissas para a implementação dos modelos:

- Balanços de massa e energia em estado estacionário

- Um único componente na fase sólida

- Distribuição discreta de partículas 


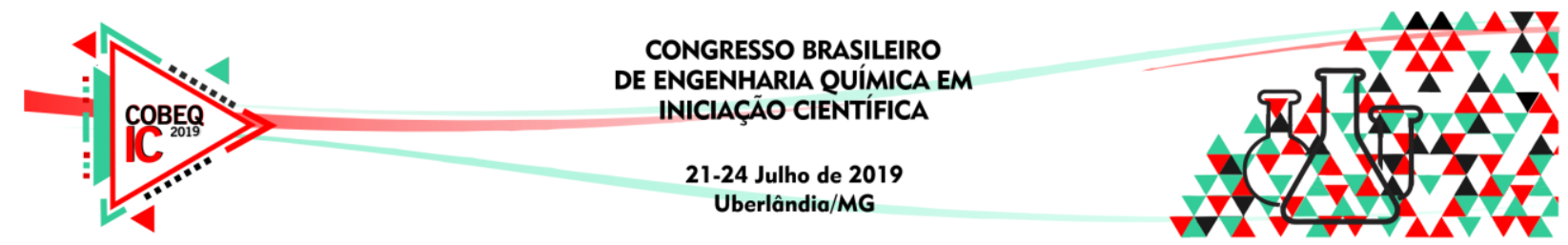

Dessa forma, por meio de Models, foram implementados modelos de correntes, fontes, sumidouros, misturadores, divisores e conversores de corrente, tanto para sistemas puramente sólidos quanto para suspensões sólido-fluido. Além disso, foram implementados os equipamentos principais, seguindo as categorias mostradas na Figura 1. Para isso, foram selecionados modelos conhecidos da literatura, como o ciclone de Lapple (1950), hidrociclone de Medronho (1984), as centrífugas de Ambler (1961) e o precipitador eletrostático de Deutsch (1922), entre outros.

Um ponto crucial para desenvolvimento de todo o trabalho foi determinar o conteúdo das correntes materiais contendo sólidos particulados. Com base nas características particulares desses sistemas e na gama de equipamentos que se objetivou implementar, as novas correntes foram definidas conforme a Figura 2 a seguir:

Figura 2 - Grandezas carregadas pelas correntes materiais nativa e implementadas.

\begin{tabular}{l}
\hline stream (nativa do EMSO) \\
•Número de componentes; \\
•Vazão molar; \\
•Temperatura; \\
•Pressão; \\
•Entalpia; \\
• Fração vaporizada; \\
-Vetor de fração molar \\
dos componentes. \\
\end{tabular}

solids_stream(nova)
-Número de classes de
partículas;
- Vazão mássica de
sólidos;
- Vetor de tamanhos de
partícula;
-Densidade das partículas;
- Vetor de frações
mássicas;
- Vetor de frações
mássicas cumulativas.

mixed_stream (nova)
- Fluid, a subcorrente de
fluido stream;
- Solids, a subcorrente de
sólidos solids_stream;
-Concentração mássica de
sólidos;
-Concentração
volumétrica de sólidos;
-Vazão volumétrica da
suspensão;
-Vazão mássica da
suspensão.

Com as correntes definidas, partiu-se para a implementação dos equipamentos. $\mathrm{O}$ grupo dos separadores sólido-sólido foi composto por: separador genérico, peneira perfeita e peneira por eficiência predefinida; O grupo dos separadores sólido-fluido foi composto por: separador genérico, separador perfeito, peneira a úmido, classificador, câmara de sedimentação, ciclone a gás, hidrociclone, centrífuga genérica, centrífuga tubular, centrífuga de discos, centrífuga de parafuso e precipitador eletrostático; $\mathrm{O}$ grupo dos filtros foi composto por: filtro genérico, filtro de discos, filtro de tambor rotativo, filtro de esteira, filtros para gases simples e rigoroso; O grupo dos cominuidores foi composto por: moinhos simples e moinhos com cálculo energético, para sólidos apenas e para sólido-fluido; Por fim, os modificadores de pressão e temperatura: aquecedor, resfriador, trocador de calor, bomba e leito fixo com escoamento incompressível. Cumpre notar que se utilizou do conceito de herança do simulador ao longo de toda a implementação: O modelo de corrente mista conta com dois submodelos em sua descrição; Os separadores sólido-fluido foram todos definidos como subtipos do modelo de separador genérico; assim como as centrífugas e filtros também foram definidos como subtipos dos modelos de centrífuga genérica e filtro genérico, respectivamente.

Paralelamente à implementação, os modelos foram testados através de FlowSheets contendo o Model a ser testado e, quando necessário, correntes materiais e energéticas. Esta etapa foi realizada a fim de depurar erros e aperfeiçoar a implementação. Posteriormente, 


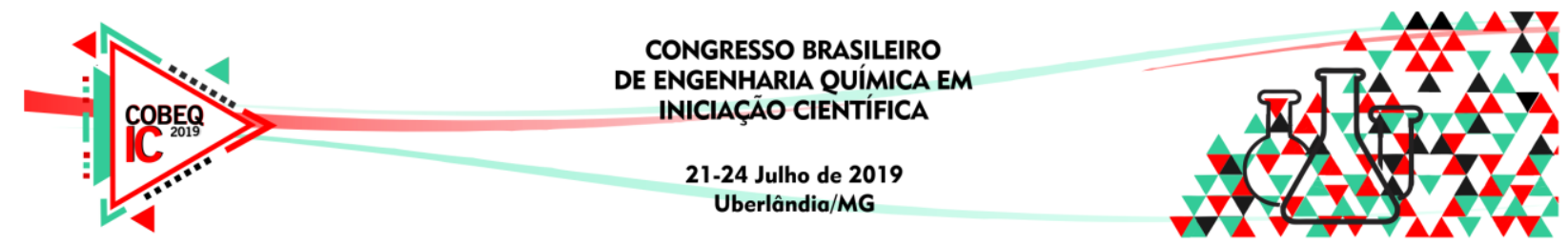

foram realizados estudos de caso simples em PFDs representando casos análogos a sistemas industriais a fim de ilustrar a utilização dos modelos.

\section{RESULTADOS E DISCUSSÕES}

\subsection{Tratamento de emissões atmosféricas}

O primeiro exemplo de processo simulado é o tratamento de uma corrente gasosa de exaustão. A corrente atravessa sequencialmente: um resfriador, um ciclone, um filtro rigoroso e um precipitador eletrostático. O diagrama na Figura 3 representa este estudo de caso.

Figura 3 - Diagrama do processo de tratamento de emissão gasosa construído no EMSO.

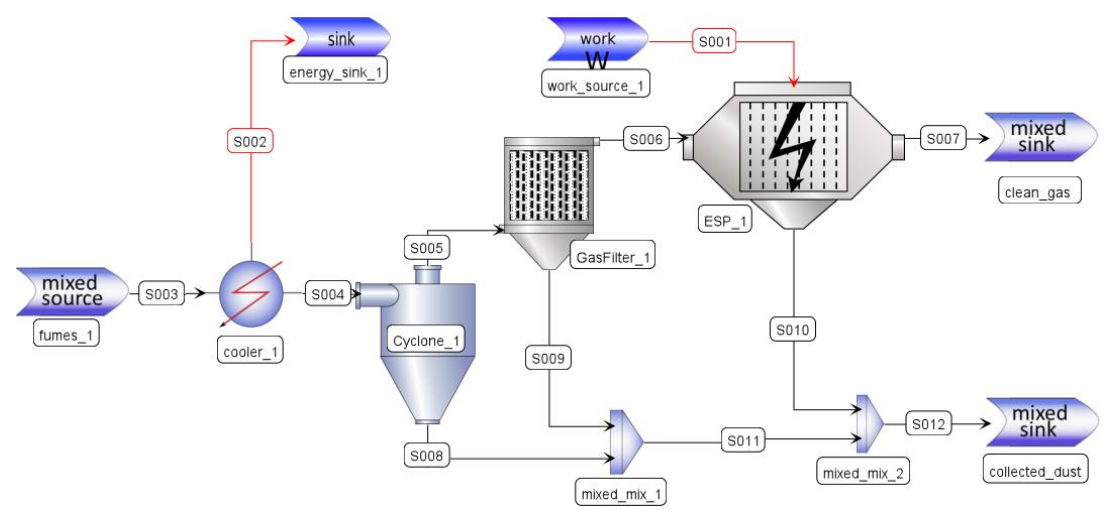

O sistema foi alimentado por $45360 \mathrm{~kg} / \mathrm{h}$ de ar a 1 bar e $1000 \mathrm{~K}$, contendo $313 \mathrm{~kg} / \mathrm{h} \mathrm{de}$ particulados de densidade $2500 \mathrm{~kg} / \mathrm{m}^{3}$. Especificou-se queda de $700 \mathrm{~K}$ na temperatura da corrente no resfriador; diâmetro de corte de 3,1 $\mu \mathrm{m}$ no ciclone de geometria Stairmand HE (Stairmand, 1951); filtro de 20 células contendo mangas de $0,8 \mathrm{~m}^{2}$ de área; precipitador eletrostático de 40 placas, com $15 \mathrm{~m}$ de comprimento e uma voltagem aplicada de $15 \mathrm{kV}$.

As distribuições de tamanhos da alimentação e das correntes de saída de cada equipamento são mostradas na Figura 4. Nota-se a diferença visual entre os perfis, com a saída do precipitador muito mais concentrada em finos em relação à alimentação.

Figura 4 - Distribuições de tamanho das partículas ao longo do processo simulado.

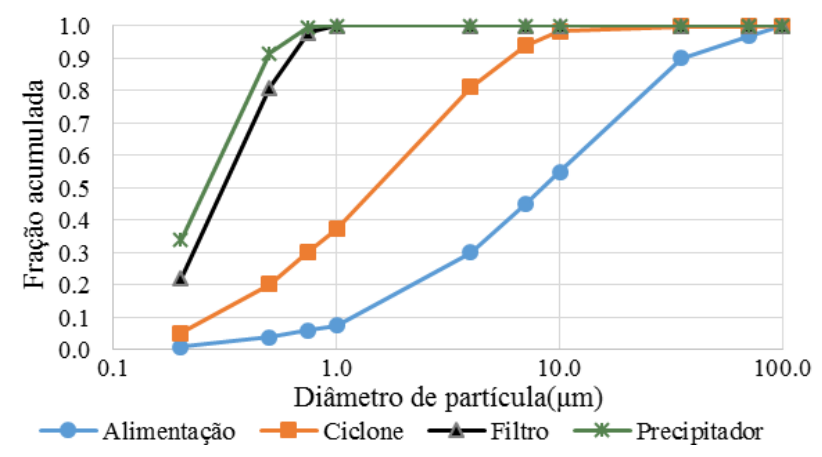

Os principais resultados dessa simulação estão resumidos na Tabela 1. Nota-se que as eficiências obtidas estão de acordo com os perfis da Figura 4 e os demais resultados são 

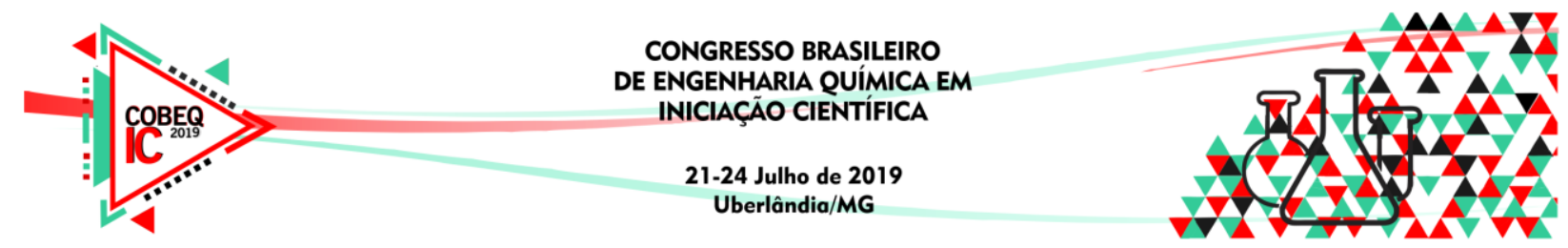

condizentes e fisicamente coerentes, além de terem sido conferidos com cálculos manuais e via planilhas eletrônicas.

Tabela 1 - Resumo dos resultados dos equipamentos do primeiro estudo de caso

\begin{tabular}{|c|l|l|l|l|}
\hline \multirow{2}{*}{ Ciclone } & Eficiência & Queda de pressão & Diâmetro & Ciclones em paralelo \\
\cline { 2 - 5 } & 0,8076 & 5,07 Torr & $1,2636 \mathrm{~m}$ & 4,62 \\
\hline \multirow{3}{*}{ Filtro } & Eficiência & Queda de pressão & Área total & Mangas por célula \\
\cline { 2 - 5 } & 0,8625 & 12,39 Torr & $111,46 \mathrm{~m}^{2}$ & 7,33 \\
\hline \multirow{2}{*}{$\begin{array}{c}\text { Precipitador } \\
\text { Eletrostático }\end{array}$} & Eficiência & Queda de pressão & Potência & Concentração \\
\cline { 2 - 5 } & 0,9303 & $3,44 \times 10^{-3}$ Torr & $1,566 \mathrm{~kW}$ & $14,145 \mathrm{mg} / \mathrm{m}^{3}$ \\
\hline
\end{tabular}

\subsection{Tratamento de minérios}

O segundo exemplo emula uma usina de moagem de minério, ilustrada na Figura 5.

Figura 5 - Diagrama do processo de tratamento de minérios construído no EMSO.

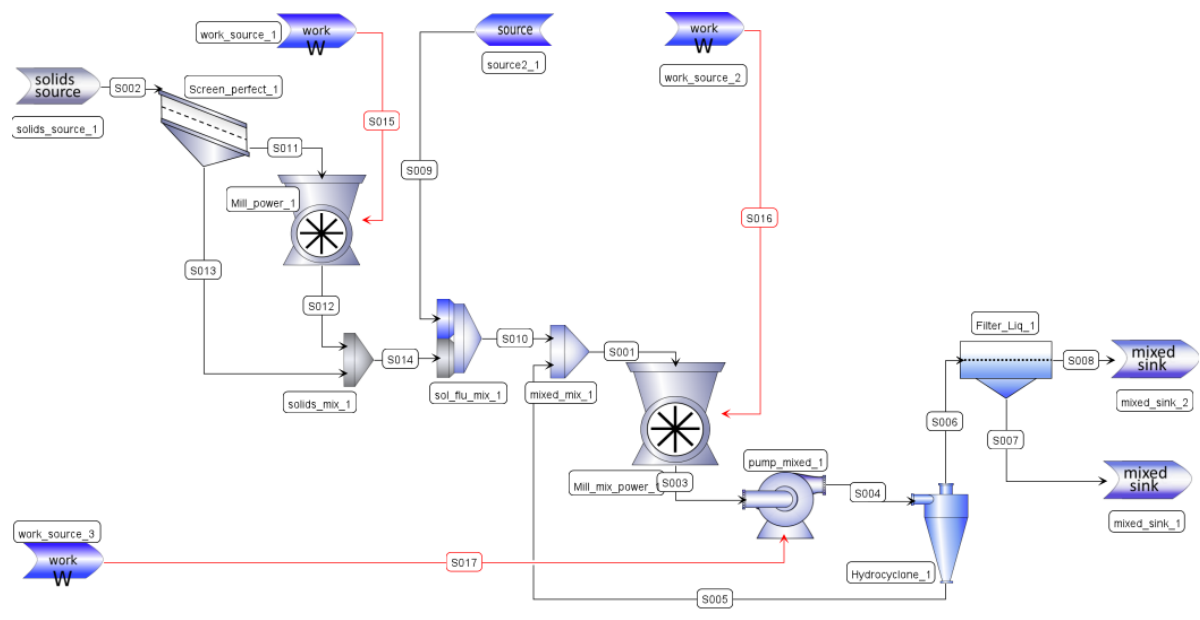

O sistema foi alimentado com $10000 \mathrm{~kg} / \mathrm{h}$ de sólidos de densidade $2500 \mathrm{~kg} / \mathrm{m}^{3}$, que passam por uma peneira de abertura $100 \mathrm{~mm}$, cujo efluente grosseiro atravessa um cominuidor. Essa corrente é misturada a $20000 \mathrm{~kg} / \mathrm{h}$ de água em condições ambientes e então é levada a um segundo cominuidor. O efluente é bombeado a um hidrociclone que recicla os grosseiros de volta ao cominuidor e descarrega os finos a um filtro, cujo produto é o minério desaguado.

O resumo dos resultados dos cominuidores é apresentado na Tabela 2.

Tabela 2 - Resumo dos resultados dos cominuidores do segundo estudo de caso

\begin{tabular}{|l|c|c|c|c|}
\hline & $\begin{array}{l}\text { Tempo de residência } \\
(\mathrm{min})\end{array}$ & $\begin{array}{l}\text { Tamanho 80\% passante } \\
\text { na entrada }(\mathrm{mm})\end{array}$ & $\begin{array}{l}\text { Tamanho 80\% passante } \\
\text { na saída }(\mathrm{mm})\end{array}$ & $\begin{array}{l}\text { Potência } \\
(\mathrm{kW})\end{array}$ \\
\hline $1^{\circ}$ cominuidor & 7,50 & 741,67 & 105,66 & 27,375 \\
\hline $2^{\circ}$ cominuidor & 8,25 & 11,68 & 5,27 & 219,199 \\
\hline
\end{tabular}




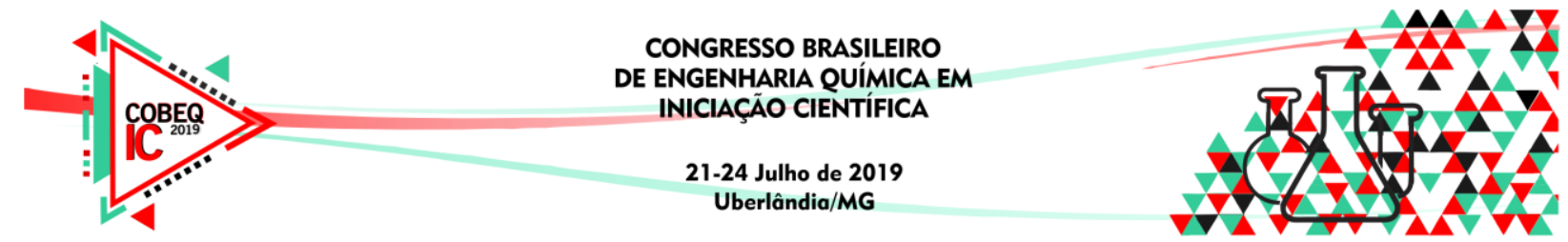

Os resultados do hidrociclone e do filtro estão resumidos na Tabela 3. Os resultados deste exemplo ilustraram o desempenho dos modelos, com valores fisicamente coerentes, conferidos por cálculos manuais e planilhas.

Tabela 3 - Resumo dos resultados do hidrociclone e do filtro do segundo estudo de caso

\begin{tabular}{|c|c|c|c|}
\hline \multicolumn{4}{|c|}{ Hidrociclone } \\
\hline Eficiência & Diâmetro de corte $(\mathrm{mm})$ & Razão de fluido & Diâmetro $(\mathrm{m})$ \\
\hline 0,73 & 2,18 & 0,17 & 1,5 \\
\hline \multicolumn{4}{|c|}{ Filtro } \\
\hline Área total $\left(\mathrm{m}^{2}\right)$ & Espessura da torta $(\mathrm{cm})$ & Tempo de filtração $(\mathrm{s})$ & Queda de pressão $\mathrm{kPa}$ \\
\hline 6,094 & 3,42 & 150 & 70 \\
\hline
\end{tabular}

\section{CONCLUSÕES}

Foi implementada uma biblioteca de modelos matemáticos para simulação, dimensionamento e otimização de processos envolvendo sólidos particulados com o simulador de processos EMSO. Os modelos implementados varrem um largo leque de aplicações e se mostraram promissores para uso futuro. Este trabalho é um passo inicial para ampliar as capacidades do simulador no que tange sólidos, e é também, ao conhecimento dos autores, a primeira ferramenta do tipo em simuladores de processos químicos gratuitos, o que pode se mostrar muito vantajoso em sua utilização pelos mais diversos usuários.

\section{REFERÊNCIAS}

AMBLER, C. Theory of Centrifugation. Ind. \& Eng. Chem., v. 53, n. 6, p. 430-433, 1961.

DEUTSCH, W. Bewegung und Ladung der Elektrizitatstrager im Zylinderkondensator. Annalen der Physik, v. 68, p. 335-344, 1922.

HARTGE, E. U.; POGODDA, M.; REIMERS, C.; SCHWIER, D.; GRUHN, G.; WERTHER, J. Flowsheet Simulation of Solids Processes. KONA Powd. and Part. Journ., v. 24, n. 24, p. 146-158, 2006.

LAPPLE, C. E. Gravity and Centrifugal Separation. Am. Ind. Hyg. Assoc. Quart., v. 11, n. 1, p. $40-48,1950$.

MEDRONHO, R. DE A. Scale-up of Hydrocyclones at Low Concentrations. University of Bradford, 1984.

SOARES, R. P.; SECCHI, A. R. EMSO: A New Environment for Modeling, Simulation and Optimization. Comp. Aid. Proc. Eng., v. 14, p. 947-952, 2003.

STAIRMAND, C. J. The design and performance of cyclone separators. Trans. of the Inst. of Chem. Eng., v. 29, p. 356, 1951. 